\title{
A INFLUENCIA DE FATORES DE PERSONALIDADE E DE ORGANIZAÇÃO DO TRABALHO NO BURNOUT EM PROFISSIONAIS DE SAÚDE
}

\section{THE INFLUENCE OF PERSONALITY AND JOB FACTORS ON BURNOUT AMONG HEALTH PROFESSIONALS}

\author{
Wilma Costa SOUZA ${ }^{1}$ \\ Angela Maria Monteiro da SILVA²
}

\begin{abstract}
RESUMO
O presente estudo investigou a relação de fatores de personalidade e de fatores ligados à organização do trabalho com a síndrome do burnout. As características de personalidade examinadas foram padrão tipo A de personalidade e traço de ansiedade. Os fatores da organização do trabalho investigados envolveram o significado do trabalho, a percepção de controle e o suporte da chefia imediata. Participaram do estudo 239 profissionais de saúde. Eles responderam a cinco medidas para avaliação de dados sócio-demográficos, personalidade tipo A, traço de ansiedade, fatores do trabalho, burnout total e suas dimensões (exaustão emocional, despersonalização e realização pessoal com o trabalho). As análises de regressão múltipla (Stepwise) indicaram que as variáveis traço de ansiedade, suporte da chefia e tipo A de personalidade foram preditores significativos do burnout total e da exaustão emocional, enquanto as variáveis traço de ansiedade e tipo $A$ de personalidade predisseram significativamente a despersonalização. Nenhuma das variáveis do estudo predisse significativamente a dimensão realização pessoal com o trabalho.
\end{abstract}

Palavras chaves: Burnout - Estresse ocupacional - Profissionais de Saúde - Fatores de personalidade - Fatores de organização do trabalho

\footnotetext{
(1) Professora do curso de graduação e pós graduação lato sensu em Fisioterapia da Universidade Gama Filho e Coordenadora do curso de pós graduação lato sensu em Fisioterapia neurofuncional da Universidade Gama Filho.

(2) Professora do curso de Mestrado em Psicologia da Universidade Gama Filho e Professora do curso de graduação em Psicologia da Universidade Federal Rural do Rio de Janeiro

ENDEREÇOPARA CORRESPONDÊNCIA:

E-MAIL : wcsrechtman@uol.com.br

Rua das Laranjeiras 28 /202, Laranjeiras. Rio de Janeiro. CEP. 22240.000 - Tel: (021) 5572413 - Cel. 91741824 AUTOR PARA ENVIO DA CORRESPONDÊNCIA : Wilma Costa Souza

INDICAÇÃO ONDE O TRABALHO FOI APRESENTADO: Dissertação submetida e aprovada pelo Programa de pós-graduação - Mestrado em Psicologia da Universidade Gama Filho como requisito parcial para obtenção do grau de Mestre em Psicologia em 16/09/99.
} 


\begin{abstract}
This study focuses the relationship between personality traits and organizational environmental factors, on one side, and burnout syndrome, on the other. The personality traits selected for this study were type A personality pattern and trait anxiety. Job factors included meaning of work, perception of control and support from supervisors. Participants were 239 health professionals. They completed five measures that assessed socio-demographic data, type A personality, trait anxiety, job factors, total burnout and its dimensions (emotional exhaustion, despersonalization and personal accomplishment). Stepwise multiple regression analyses indicated that trait anxiet, support from supervisors and type A personality were significant predictors of total burnout and emotional exhaustion. In addition, trait anxiet and type A personality significantly predicted despersonalization. However, there were no significant predictors of personal accomplishment.
\end{abstract}

Key words: Burnout - Occupational stress - Health professionals - Personality traits - Organizational environmental factors

\section{INTRODUÇÃO}

O estudo dos fatores que levam ao estresse e a influência do mesmo na gênese das doenças em geral tem recebido grande ênfase por parte dos pesquisadores. Entretanto, a investigação do trabalho como fator desencadeante do estresse só mais recentemente tem recebido atenção.

Dentre os diversos modelos de estresse ocupacional, optou - se no presente estudo pelo modelo do Burnout, conforme desenvolvido por Maslach \& Jackson (Maslach, 1976).

A síndrome atinge principalmente profissionais que atuam na área de ciências humanas tais como enfermeiros, fisioterapeutas, médicos e assistentes sociais, caracterizando - se por uma reação de estresse crônico (Reinhold, 1996).

A síndrome do burnout talvez possa oferecer uma explicação para as dificuldades percebidas na relação profissional de saúde - paciente, dificuldades estas que, ao mesmo tempo em que não contribuem para a recuperação dos doentes, podem levar ao sentimento de grande insatisfação com o trabalho muitas vezes referido pelos profissionais. Na síndrome do burnout é descrita a dificuldade do profissional em lidar com as emoções de seus pacientes levando o a tratá - los de forma impessoal e desumanizada (Maslach, 1976). Neste caso, o profissional de saúde pode utilizar-se de estratégias negativas para enfrentar a situação, distanciando - se de seus pacientes e passando a encará - los como algo totalmente destituído de qualidades humanas.

O burnout parece acometer pessoas altamente motivadas e dedicadas, observando -se nos profissionais acometidos uma queda na performance que influi na qualidade dos serviços prestados. A síndrome se correlaciona com insônia, aumento do uso de álcool e drogas, problemas no casamento e na família (Maslach \& Schaufeli, 1993).

A síndrome do Burnout foi inicialmente descrita em 1974 por Frendenberg (França,1987). O termo pode ser traduzido como aquilo que deixou de funcionar por exaustão de energia e foi usado pelo autor para designar uma resposta dos indivíduos ao estresse ocupacional. Ainda na década de 70, Maslach \& Jackson passaram a investigar a síndrome, desenvolvendo cientificamente um 
modelo de burnout. Os autores também elaboraram o Maslach Burnout Inventory para medir a síndrome, sendo este instrumento atualmente usado na maioria das pesquisas sobre o tema (Maslach \& Schaufeli, 1993).

Schaufeli, Maslach \& Marek (1993) conceituam o burnout como síndrome de exaustão, despersonalização e baixa realização pessoal com o trabalho, que pode ocorrer com indivíduos que trabalham para pessoas, especialmente para as que têm algum tipo de problema. A exaustão refere-se ao sentimento de sobrecarga emocional e de esgotamento e é a dimensão que mais se aproxima de uma variável de estresse. A despersonalização, por outro lado, constitui um novo constructo que não aparece diretamente na literatura sobre o estresse (Cox, Kuk \& Leiter, 1993). O profissional afetado pelo burnout, não é mais capaz de lidar com as emoções das pessoas que atende e começa a tratá - las de forma desumanizada (Maslach ,1976).

A terceira dimensão proposta pela autora, é a da baixa realização pessoal com o trabalho que se expressa através da tendência do indivíduo a se avaliar negativamente em relação a seu desempenho.

A síndrome, então, constitui-se em um modelo multidimensional onde a ocorrência de um componente pode precipitar o desenvolvimento dos outros dois (Golembiewsky, Scherb \& Boudreau, 1993) ou pode ocorrer que os componentes se desenvolvam ao mesmo tempo, visto tratarem-se de reações a diferentes aspectos do ambiente de trabalho como afirma Leiter (1993).

É característico na síndrome um sentimento de desilusão e frustração que ocorre em pessoas que possuíam grandes expectativas em relação às suas carreiras. É resultado, então, de um processo de desilusão onde o trabalhador percebe que não consegue retirar de seu trabalho um sentido, um significado existencial (Pines, 1993).

Entre as características de personalidade que favorecem o desenvolvimento do burnout destacam-se a motivação, o entusiasmo, a dedicação ao trabalho e a tendência ao perfeccionismo (Squires \& Livesley, 1984).

Segundo França (1987), os profissionais atingidos pela síndrome são pessoas que mergulham fundo em seu trabalho, não sabem dizer não, se ocupam com várias coisas ao mesmo tempo e têm compulsão para o trabalho, retirando dele grande parte de sua satisfação pessoal. Paradoxalmente, estas características são as mais valorizadas pelos departamentos de seleção de pessoal. O profissional que não mede sacrifícios pela empresa e tem grande necessidade de vencer e ser reconhecido, dificilmente relaxa e se, além disso, mostra pouca habilidade para lidar com o estresse em situações interpessoais pode ser levado ao burnout (Reinhold, 1996).

Dentre os critérios diagnósticos do burnout destacam-se um estado geral de fadiga acompanhado de perda da auto - estima resultante de um sentimento de incompetência profissional e insatisfação com o trabalho (Maslach \& Schaufeli, 1993). Os mesmos autores apontam como principal indicador uma diminuição significativa da performance no trabalho.

O presente estudo não poderia ser abrangente a ponto de investigar todos os aspectos envolvidos no estresse ocupacional. Desta forma, optou-se pelo estudo da influência de fatores intrínsecos à personalidade e de estressores ligados à organização do trabalho na síndrome de burnout.

Considerando-se os diversos aspectos ligados à personalidade, optou-se pelas variáveis padrão de personalidade tipo $\mathrm{A} \mathrm{e}$ traço de ansiedade. Em relação aos fatores ligados à organização do trabalho, selecionou-se, o significado do trabalho, o 
sentimento de controle e o suporte oferecido pela chefia imediata.

O padrão de personalidade tipo A pode ser definido como um estilo de vida caracterizado por extrema competitividade, luta por realização, agressividade, pressa, impaciência, inquietação, vigilância, fala explosiva, tensão da musculatura facial e sentimento de estar sob pressão do tempo (Cooper, 1987).

O traço de ansiedade refere-se a diferenças individuais relativamente estáveis em propensão à ansiedade, isto é , a diferença na tendência de reagir a situações percebidas como ameaçadoras com elevações de intensidade no estado de ansiedade (Biaggio, Natalício \& Spielberg, 1977).

O significado do trabalho pode ser entendido como a relevância percebida pelo sujeito acerca do trabalho que executa, avaliando o seu grau de importância para a sociedade. Quanto ao sentimento de controle, ele reflete a percepção do indivíduo acerca da sua autonomia em relação à divisão e organização das tarefas que ele executa. Por fim, o suporte oferecido pela chefia refere-se à percepção do sujeito quanto a orientação recebida para a realização das suas tarefas, adequação da supervisão e possibilidade de obter ajuda efetiva em relação às dificuldades que encontra no desenvolvimento do seu trabalho

O presente estudo visou investigar se fatores de personalidade (traço de ansiedade e padrão tipo $A$ ) e fatores da organização do trabalho ( significado do trabalho, percepção de controle e suporte da chefia) são preditores do Burnout e suas dimensões.

\section{METODOLOGIA}

\section{Participantes}

A pesquisa foi realizada em uma amostra de 239 profissionais da área de saúde, de nível superior e de diversas categorias profissionais: médicos, fisioterapeutas, psicólogos, assistentes sociais, dentistas e enfermeiros.

Os participantes eram trabalhadores de instituição pública, privada, autônomos ou empregadores e exerciam suas funções em ambulatórios, enfermarias, emergências, ou ainda, prestavam atendimento domiciliar.

Foram pesquisados profissionais do sexo feminino (81\%) e masculino (19\%), na faixa etária de 22 a 72 anos. A média e desvio padrão da idade em anos foram $39,10+10,00$ (média + DP).

A maioria da amostra foi constituída por médicos $(32,2 \%)$ e fisioterapeutas $(29,7 \%)$.

\section{Instrumentos}

Os níveis de ansiedade traço foram operacionalizados pela escala de ansiedade traço do Inventário de Ansiedade Traço - Estado (IDATE), elaborado por Spielberg e colaboradores e adaptado para a população brasileira por Biaggio e colaboradores (1977). A escala de ansiedade - traço contém 20 afirmativas que descrevem como os sujeitos geralmente se sentem. Para cada item os sujeitos escolhem uma das quatro opções de resposta, que variam de quase nunca a quase sempre. Os escores totais da escala variam de 20 a 80 , considerando-se que quanto maior o escore, maior o grau de ansiedade do indivíduo.

A síndrome do Burnout foi operacionalizada através do Maslach Burnout Inventory (MBI) desenvolvido por Maslach \& Jackson (1981) e adaptado para população brasileira por Carvalho (1995). Este instrumento consta de três subescalas que foram elaboradas para abranger as três dimensões da síndrome, isto é, exaustão emocional, despersonalização e realização pessoal. O MBI, adaptado por Carvalho (1995) compõe-se de 22 itens, sendo 9 itens para medir a exaustão emocional, 8 itens para a 
realização pessoal com o trabalho e 5 itens para a despersonalização. O cálculo dos escores dos indivíduos é realizado através da atribuição de valores relativos à freqüência. Para cada item há seis opções de respostas que variam de muitas vezes ao ano a todos os dias. O escore total bruto dos sujeitos é obtido através do somatório dos pontos de cada item do inventário. O escore do sujeito em cada uma das dimensões (exaustão, realização pessoal e despersonalização) também é computado pelo somatório dos pontos dos itens relativos a cada uma das dimensões.

O padrão de personalidade tipo A foi mensurado através de instrumento anteriormente empregado por Malagris (1992) para amostras brasileiras. O instrumento é composto de uma escala contendo 10 afirmativas. Os sujeitos assinalam cada item da escala considerando-o verdadeiro ou falso. Os escores variam potencialmente de 0 a 10. Quanto mais elevado o escore total mais o indivíduo apresenta o padrão de personalidade tipo A.

O significado do trabalho, o sentimento de controle e o suporte oferecido pela chefia foram avaliados através de itens especialmente concebidos para este fim. Em trabalhos prévios, estas variáveis têm sido medidas em escalas de 2 pontos (sim /não), como em Rego (1993) e Carvalho (1995). No presente estudo optou se por escalas analógicas visuais que permitem uma medida mais completa das variáveis. $\mathrm{Na}$ escala analógica visual os sujeitos avaliaram cada variável colocando um "X" numa linha de $10 \mathrm{~cm}$ que possuía rótulos em cada um dos extremos. Para computar os escores usou-se uma régua medindo a distância do ponto inicial ao ponto assinalado pelo " $X$ " que o sujeito marcou. Quanto mais elevado o escore, maior o significado do trabalho, a percepção de controle ou o suporte oferecido pela chefia.

As variáveis sócio - demográficas de possível relevância foram avaliadas através de um questionário especialmente construído para esta finalidade.

\section{Procedimento}

Os participantes da pesquisa foram contatados em seus respectivos locais de trabalho, ou seja, hospitais da rede pública e privada ou clínicas particulares. Foram informados dos objetivos da pesquisa e do sigilo em relação às respostas. Os que concordaram em participar receberam os questionários e foram orientados quanto ao preenchimento.

\section{Análises Estatísticas}

Empregou-se Análise de Variância Simples para verificar se homens e mulheres diferiam significativamente nas variáveis do estudo, e para examinar se os sub grupos com diferentes tempo de profissão diferiam significativamente nessas variáveis.

Por fim, produziu-se análises de regressão múltipla (método Stepwise) para examinar se a personalidade tipo $\mathrm{A}$, o traço de ansiedade, o significado do trabalho, o controle percebido e o apoio da chefia, em conjunto, predizem significativamente o burnout total e suas dimensões. Em todas as análises usou-se um nível de significância igual a 0,05.

\section{Resultados}

Os resultados obtidos a partir da aplicação de testes (ANOVA one way), tendo a variável sexo e tempo de profissão como intervenientes e as demais variáveis como básicas do estudo, indicaram que não existem diferenças significativas ao nível de $5 \%$ entre os valores médios das variáveis analisadas (Tabelas 1 e 2).

Desta forma, foi possível admitir um único grupo para o estudo independentemente dos parâmetros sexo e tempo de profissão. 
Tabela 1. Comparações das médias de homens e mulheres nas variáveis do estudo.

\begin{tabular}{|l|c|c|}
\hline Variável & F calculado & Significado $\mathbf{p}$ \\
\hline Idade & 2.218 & 0.1377 \\
\hline Significado do Trabalho & 0.156 & 0.6976 \\
\hline Sentimento de Controle & 0.449 & 0.5108 \\
\hline Apoio da Chefia & 0.611 & 0.4434 \\
\hline Padrão Tipo A & 1.082 & 0.2994 \\
\hline Traço de ansiedade & 1.098 & 0.2959 \\
\hline Exaustão emocional & 0.731 & 0.4025 \\
\hline Realização pessoal & 0.112 & 0.7418 \\
\hline Despersonalização & 2.998 & 0.0847 \\
\hline Carga Horária & 2.116 & 0.1471 \\
\hline Tempo de Profissão & 2.068 & 0.1517 \\
\hline
\end{tabular}

As médias observadas serão diferentes quando $p<0,05$

Tabela 2. Comparações das médias das diversas categorias de tempo de profissão para as variáveis do estudo.

\begin{tabular}{|l|c|c|}
\hline Variável & F calculado & Significado p \\
\hline Idade & 120.60 & 0.0001 \\
\hline Significado do Trabalho & 0.926 & 0.4650 \\
\hline Sentimento de Controle & 0.903 & 0.4800 \\
\hline Apoio da Chefia & 1.267 & 0.2789 \\
\hline Padrão Tipo A & 1.584 & 0.1659 \\
\hline Traço de ansiedade & 1.464 & 0.2027 \\
\hline Exaustão emocional & 1.503 & 0.1896 \\
\hline Realização pessoal & 0.971 & 0.4365 \\
\hline Despersonalização & 0.272 & 0.9281 \\
\hline Carga Horária & & \\
\hline
\end{tabular}


A primeira análise de regressão múltipla produzida, tendo como variável dependente o burnout total e como variáveis preditoras os fatores de personalidade e de organização do trabalho, indicou que três preditores alcançaram significância ao nível de 5\% : traço de ansiedade, apoio da chefia e personalidade tipo A (tabela 3 ). O coeficiente de determinação, $R^{2}$, foi 0,349 , apontando que $35 \%$ da variância do burnout total pode ser explicada através de seu relacionamento com os três preditores.
Na segunda análise de regressão múltipla considerou - se como variável dependente a exaustão emocional e como preditores os fatores de personalidade e de organização do trabalho, e três preditores alcançaram significância ao nível de 5\%: traço de ansiedade, apoio da chefia imediata e personalidade tipo $\mathrm{A}$ (tabela 4). O coeficiente de determinação, $R^{2}$, foi 0,33 , indicando que $33 \%$ da variância da exaustão pode ser explicada através do seu relacionamento com os três preditores.

Tabela 3. Resultado significativos da primeira análise de repressão múltipla, tendo o burnout total como VD

\begin{tabular}{|l|c|c|c|}
\hline Preditor & Coeficiente de Regressão & $\mathbf{t}$ & Significância \\
\hline Traço Ansiedade & 1,01 & 7,27 & $\mathrm{P}=0,000$ \\
\hline Apoio Chefia & $-1,64$ & $-3,95$ & $\mathrm{P}=0,000$ \\
\hline Tipo A & 1,56 & 2,11 & $\mathrm{P}=0,036$ \\
\hline
\end{tabular}

Tabela 4. Resultado significativos da segunda análise de repressão múltipla, em que a exaustão foi a VD

\begin{tabular}{|l|c|c|c|}
\hline Preditor & Coeficiente de Regressão & $\mathbf{t}$ & Significância \\
\hline Traço Ansiedade & 0,71 & 7,43 & $\mathrm{P}=0,000$ \\
\hline Apoio Chefia & $-0,98$ & $-3,41$ & $\mathrm{P}=0,001$ \\
\hline Tipo A & 1,11 & 2,18 & $\mathrm{P}=0,031$ \\
\hline
\end{tabular}

Uma terceira análise de regressão múltipla foi produzida. A variável dependente foi a despersonalização, e os preditores foram padrão tipo $A$, traço de ansiedade, significado do trabalho, controle e apoio da chefia imediata. Os resultados mostraram que dois preditores foram significativos: traço de ansiedade e padrão de personalidade tipo $\mathrm{A}$ (tabela 5). $\mathrm{O}$ coeficiente de determinação, $R^{2}$, foi 0,13 mostrando que $13 \%$ da variância da despersonalização pode ser explicada através de seu relacionamento com os dois preditores.

A quarta análise de regressão múltipla teve como variável dependente a realização pessoal e como preditores os fatores de personalidade e de organização do trabalho. Os resultados indicaram que nenhum preditor alcançou significância ao nível de 5\%.Uma análise de regressão múltipla padrão confirmou estes resultados (tabela 6). 
Tabela 5. Resultado significativos da terceira análise de repressão múltipla, cuja a VD foi a despersonalização.

\begin{tabular}{|l|c|c|c|}
\hline Preditor & Coeficiente de Regressão & t & Significância \\
\hline Traço Ansiedade & 0,13 & 4,18 & $\mathrm{P}=0,000$ \\
\hline Tipo A & 0,39 & 2,27 & $\mathrm{P}=0,024$ \\
\hline
\end{tabular}

Tabela 6. Resultado significativos da quarta análise de repressão múltipla, que teve como VD a realização pessoal.

\begin{tabular}{|l|c|c|c|}
\hline Preditor & Coeficiente de Regressão & $\mathbf{t}$ & Significância \\
\hline Traço Ansiedade & 0,15 & 1,72 & $\mathrm{P}=0,08$ \\
\hline Tipo A & 0,24 & 0,54 & $\mathrm{P}=0,59$ \\
\hline Significado & $-1,04$ & $-1,61$ & $\mathrm{P}=0,11$ \\
\hline Controle & 0,67 & 1,80 & $\mathrm{P}=0,07$ \\
\hline Apoio Chefia & $-0,43$ & $-1,69$ & $\mathrm{P}=0,09$ \\
\hline
\end{tabular}

\section{DISCUSSÃo}

A amostra foi composta de 239 sujeitos sendo a maioria $(80,8 \%)$ do sexo feminino sugerindo uma nítida preferência deste sexo por profissões da área de saúde.

A aplicação de testes (ANOVA one way), indicou que as variáveis sexo e tempo de profissão não interferiram significativamente nas variáveis do estudo. No entanto, as variáveis idade e tempo de profissão se correlacionaram significativamente, o que já era esperado, ou seja, quanto maior a faixa etária, maior o tempo de profissão.

Os resultados aqui obtidos foram diferentes dos encontrados por Maslach \& Jackson (1981) em relação ao sexo. Os autores apontam que mulheres apresentam escores mais elevados que homens para exaustão emocional enquanto estes últimos apresentam maior tendência a despersonalização. A dupla jornada de trabalho a que as mulheres geralmente são submetidas é apontada por Cushway \& Tyler (1996) como fator que contribuiria para maiores níveis de exaustão emocional. Diferenças culturais talvez expliquem a discrepância entre os resultados obtidos no presente estudo e aqueles relatados por Maslach \& Jackson (1981). A maior possibilidade de delegar a execução de tarefas domésticas a outras mulheres, como ocorre na classe média brasileira, provavelmente diminui o impacto da dupla jornada. $\mathrm{Na}$ investigação da síndrome de burnout em professores do ensino médio paulista, Rego (1993) também não encontrou diferenças significativas entre homens e mulheres quanto ao burnout.

A idade e o tempo de profissão são relacionadas aos níveis de burnout total e suas dimensões em diversos estudos (Maslasch \& Jackson, 1981; Ullrich \& 
Fitzgerald, 1990; Cushway \& Tyler, 1996; Moore \& Cooper 1996). De modo geral, observou-se que o burnout é mais acentuado nos primeiros anos de profissão já que os profissionais tendem a se sentir mais inseguros em relação a seus conhecimentos, são mais afetados pelas reações de seus pacientes e, muitas vezes, nutrem falsas expectativas sobre suas carreiras. Profissionais com mais tempo de profissão, provavelmente já desenvolveram estratégias de coping que Ihes permitem lidar melhor com as situações estressantes advindas do trabalho. Segundo Moore \& Cooper (1996), os profissionais com maior tempo de carreira têm menor carga horária semanal, trabalham mais em tarefas administrativas e gerenciais e dedicam-se menos ao atendimento direto de pacientes, o que poderia contribuir para menores níveis de burnout .

Os resultados encontrados no presente estudo apontaram que os grupos com diferentes tempos de profissão não diferiram significativamente quanto ao burnout total ou suas dimensões, contradizendo, portanto, os resultados de Moore \& Cooper (1996). Os profissionais de saúde que integram a amostra da presente pesquisa, provavelmente devido a aspectos sócio - culturais, diferem dos participantes americanos descritos no estudo de Moore \& Cooper (1996). No presente estudo, apesar da média de idade (39 anos) apontar para um tempo de profissão razoável, a média da carga horária trabalhada semanalmente (46 h) encontra-se acima da preconizada pela Constituição Federal Brasileira (44 horas semanais). Possivelmente, os baixos salários e a precarização das relações trabalhistas têm levado os profissionais com mais tempo de profissão a jornadas mais longas e diversos vínculos empregatícios. Quanto aos mais jovens, as oportunidades oferecidas pelo mercado de trabalho apontam para jornadas longas, extenuantes física e mentalmente e sem vínculo formal. Uma pesquisa realizada sobre o perfil do médico no Brasil (Machado,1996) demonstra esta realidade.
Essas condições desfavoráveis restringem atividades fora do trabalho sejam elas sociais, culturais ou desportivas. A atual situação sócio - econômica do país pode estar levando os profissionais de saúde a um excesso de trabalho e, consequentemente, afetando as atividades de lazer. O lazer é considerado importante mediador do estresse, podendo promover a satisfação das necessidades psicológicas individuais, favorecer o bem estar físico e mental e gerar crescimento pessoal (Catalbianco, 1995). Neste sentido, a conscientização dos trabalhadores sobre a necessidade de se dedicarem, sempre que possível, a atividades de lazer poderia contribuir para menores níveis de estresse e maior satisfação com o trabalho.

O padrão de personalidade tipo A mostrou-se um preditor significativo do burnout total, da exaustão emocional e da despersonalização.

A relação entre aspectos da personalidade e a síndrome de burnout não tem sido muito investigada. Schaufeli e cols. recomendam que "seja desenvolvido um modelo conceitual que hipotetize quais variáveis de personalidade seriam relevantes no desenvolvimento da síndrome do burnout " (Schaufeli, Maslasch \& Marek, 1993, p. 30).

De modo geral, considera-se que os indivíduos tipo A possuem um forte senso de urgência, o que os leva a tentar realizar mais e mais tarefas em cada vez menos tempo. Apresentam grande sentimento de hostilidade que se traduz por raiva e irritação e demonstram grande ambição e competitividade. Dentre os diversos aspectos que integram o padrão tipo A, a hostilidade é considerada o fator mais significativo no desenvolvimento do estresse (Hockenbury \& Hockenbury, 1997).

No presente estudo constatou-se que o traço de ansiedade é um preditor significativo do burnout e todas as suas dimensões, exceto a realização pessoal com o trabalho.

Os resultados encontrados sugerem que os indivíduos com alto traço de ansiedade são 
mais vulneráveis ao estresse ocupacional e ao burnout e, portanto, poderiam ser beneficiados por um programa de manejo do estresse.

Diversos programas de manejo de estresse surgiram nos últimos anos com o objetivo de ensinar aos indivíduos como apreender eventos estressantes e como desenvolver estratégias adequadas de coping. $\mathrm{O}$ treinamento de técnicas de relaxamento e meditação tem sido empregado por diversas empresas com o objetivo de diminuir o estresse de seus empregados. A meditação pode diminuir o consumo de oxigênio e os batimentos cardíacos reduzindo a ativação fisiológica do organismo e a ansiedade (Hockenbury \& Hockenbury, 1997).

Lipp, Romano, Covolan \& Nery (1990, p. 149) propõem um tratamento comportamental para o estresse composto de quatro pilares: "i) alimentação anti - estresse buscando suprir o organismo com nutrientes gastos em momentos em que uma adaptação maior lhe é exigida, ii) relaxamento para restaurar o estado de homeostase quebrado em momentos de estresse, iii) exercícios físicos capazes de levar o organismo a produzir endorfinas com o propósito de promover uma sensação de tranqüilidade e bem estar, iv) tratamento comportamental que inclui a reestruturação de crenças irracionais, treino em assertividade e em controle da ansiedade, aquisição de técnicas de resolução de problemas e manejo do tempo, além de prática na percepção e no controle do padrão tipo A de comportamento ".

Vários pesquisadores e teóricos como por exemplo Auerbach \& Gramlig (1998) declaram que fatores de personalidade como traço de ansiedade e padrão tipo A podem ser reduzidos, mas seriam necessários vários anos de psicoterapia. No entanto, a médio prazo, o emprego do relaxamento, da meditação e da auto - sugestão poderiam ajudar a minimizar a influência destes fatores.

Em relação às medidas acima descritas para a redução do estresse, é importante ressaltar que estas são centradas no próprio sujeito sem a intenção de influir diretamente no ambiente estressor. Desta forma, corre-se o risco de atribuir ao trabalhador a inteira responsabilidade no manejo de seu estresse, limitando-se assim a ação de cunho mais político e social que envolva a mudança do ambiente e condições do trabalho.

No presente estudo o apoio da chefia foi preditor do burnout total e da exaustão emocional. O apoio ou suporte da chefia foi relacionado ao burnout em diversos outros estudos. Hillhouse \& Adler (1997) demonstraram menores níveis de burnout em enfermeiras que percebiam maior suporte de suas chefias. Para os autores, o suporte promove o senso de competência, a auto - eficácia e a auto - estima.

Conclui-se que o baixo apoio da chefia tende a favorecer o desenvolvimento do burnout. Por outro lado, o oferecimento de apoio pela chefia parece diminuir a chance do profissional apresentar burnout.

Medidas de moderado impacto na estrutura organizacional tais como: instalações para realização de exercícios, delegação de responsabilidades que fortaleçam o controle, e suporte adequado oferecido pela chefia são descritas como capazes de contribuir para menores níveis de estresse (Härtl, 1999).

Em relação ao apoio da chefia, preditor da burnout total e da exaustão, a conscientização e treinamento dos chefes, poderiam levá-los ao oferecimento de suporte adequado aos profissionais de saúde.

Por fim, futuros estudos podem esclarecer a natureza do suporte social melhor indicado para as diversas categorias de profissionais de saúde, considerando-se suas diferentes funções.

Uma importante função da pesquisa em psicologia social envolve avaliar cientificamente os fenômenos possibilitando, assim, sua veiculação na sociedade. Desta forma, contribui-se para a formulação de propostas que possam alterar a realidade descrita. 
Em relação aos profissionais de saúde, a discussão dos diferentes aspectos do burnout (causas, conseqüências e meios para a prevenção) deveria permear nos cursos de graduação, nas associações e nos sindicatos de classe permitindo aos profissionais maior compreensão, identificação e prevenção do problema. Empenhados na arte de prevenir e curar doenças, estes profissionais nem sempre têm a noção de seu próprio adoecer no trabalho.

\section{REFERÊNCIAS BIBLIOGRÁFICAS}

AUERBACH, S.M. \& Gramling, S.E. (1998). Stress management Psychological foundations. New Jersey : Prentice Hall.

BIAGGIO, A.M.B.; Natalício, L.; Spielberg, C.D. (1977). Desenvolvimento da forma experimental em português do inventário de ansiedade traço - estado (IDATE). Arquivos Brasileiros de Psicologia Aplicada, 29, $31-42$.

CARVALHO, M.M.B. (1995). O professor, um profissional, sua saúde e a educação em saúde na escola. Tese de Doutorado. Faculdade de Saúde Pública da Universidade de São Paulo.

CATALBIANCO, M. R. (1995). Main and stress - moderating health benefits of leisure. Loisir \& Société.18, 33 - 52.

COOPER, C.L. (1987). Stress prone behaviour: type A pattern. Em Kalimo, R; Bataw, M.A.E \& Cooper, C.L. (Orgs). Psychosocial factors at work and their relation to health.(pp 134 - 137). Genebra: World Health Organization.

COX, T; KuK, G \& Leiter, M. (1993). Burnout, health, work stress, and organizational healthness . Em Schaufeli, W.B; Maslach, C \& Marek, T. (Orgs). Professional burnout, recent developments in theory and research (pp 177 - 193). Philadelphia: Taylor \& Francis.
CUSHWAY, D \& Tyler, P. (1996). Stress in clinical psychologists. International Journal of Social Psychiatry, 42 . 141 -149.

FRANÇA, H.H. (1987). A síndrome do burnout. Revista Brasileira Médica, 44, 25-27.

GOLEMBIEWSKY, R.T; Scherb, K\& Boudreau, R.A. (1993). Burnout in cross - national settings: generic and model - specific perspectives. Em Schaufeli, W.B; Maslach, C \& Marek, T.(Orgs). Professional burnout, recent developments in theory and research. (217-236).Philadelphia : Taylor \& Francis.

HÄRTL, G .(1999). Control de las fuentes de estrés en grupos de alto riesgo. Revista da Oficina Internacional do Trabalho,18, $1-6$.

HILLHOUSE, J.J \& Adler, C.M. (1997). Investigating stress effects patterns in hospital staff nurses : results of a cluster analysis. Social Science and Medicine, 45, 1781 - 1788.

HOCKENBURY, D.H. \& Hockenbury, S.E. (1997). Psychology. New York: Worth Publishers.

LEITER, M.P. (1993). Burnout as a developmental process: consideration of models. Em Schaufeli, W.B ; Maslach, C \& Marek, T.(Orgs). Professional burnout, recent developments in theory and research (pp 237 - 250). Philadelphia : Taylor \& Francis.

LIPP, M.E.N; Romano, A .N.S.F; Covolan, M.A; Nery, M.J.G.S (1990). Como enfrentar o stress. São Paulo : Ícone.

MACHADO, M. H. (1996). Perfil dos médicos no Brasil, análise preliminar. Rio de Janeiro Fiocruz.

MALAGRIS, L.E.N. (1992). Stress e úlceras gastroduodenais - interações clínico psicológicas. Dissertação de mestrado. Departamento de Pós Graduação em 
Psicologia do Instituto de Psicologia da PUC de Campinas.

MASLACH, C. (1976). Burned - out. Human Behaviour, 5, 16 - 22.

MASLACH, C \& Jackson, S. (1981). The measurement of experienced burnout. Journal of Occupational Behaviour, 2, $99-113$.

MASLACH, C \& Schaufeli, W.B. (1993). Historical and conceptual development of burnout. Em Schaufeli, W.B; Maslach, C \& Marek, T. (Orgs). Professional burnout recent developments in theory and research. (pp 1 - 16 ) Philadelphia: Taylor \& Francis.

MOORE, K.A \& Cooper, C.L. (1996). Stress in mental health professionals: a theoretical overview. International Journal of Social Psychiatry, 4282 - 89.

PINES, A. M. (1993). Burnout: an existential perspective. Em Schaufeli, W.B ; Maslach, C \& Marek, T.(Orgs), Professional burnout, recent developments in theory and research (pp 33 - 51). Philadelphia: Taylor \& Francis.

REGO, M.P.C.M.A. (1993). Trabalho hospitalar e saúde mental, o caso de um hospital geral e público no município do Rio de Janeiro. Dissertação de mestrado. Instituto de Medicina Social do Centro Biomédico da Universidade do Rio de Janeiro.

REINHOLD, H.H. (1996). Stress ocupacional do professor. Em Lipp, M.E.N. (Org), Pesquisa sobre stress no Brasil. (pp. 169 - 194).São Paulo: Papirus.

SCHAUFELI, W.B, Maslach, C \& Marek, T. (1993). The future of burnout. Em Schaufeli, W.B; Maslach, C \& Marek, T. (Orgs), Professional burnout, recent developments in theory and research (pp 253 - 259). Philadelphia: Taylor \& Francis.

SQUIRES, A \& Livesley, B. (1984). Beware of burnout. Journal of the Chartered Society of Physiotherapy, 70 , 235 - 238.

ULLRICH, A. \& Fitzgerald, P. (1990). Stress experienced by physicians and nurses in the cancer ward. Social Science and Medicine, 31, 1013 - 1022. 